\title{
Construction and Performance of the sTGC and MicroMegas chambers for ATLAS NSW Upgrade
}

Givi Sekhniaidze

INFN sezione di Napoli

On behalf of ATLAS NSW community

14th Topical Seminar on Innovative Particle and Radiation Detectors (IPRD16) 3 - 6 October 2016 Siena, Italy 


\section{Outline}

- Motivation for ATLAS New Small Wheel upgrade

- Detector technologies and New Small Wheel layout

- sTGC:

- Detector structure and construction

- Module-0 construction

- Test beam results at FermiLab and CERN

- MicroMegas:

- MicroMegas design and challenges

- Module-0 construction

- Test beam results at CERN 


\section{Motivation}

The instantaneous luminosity of the Large Hadron Collider at CERN will be increased up to a factor of five to seven with respect to the design value by undergoing an extensive upgrade program over the coming decade.

Add triggering capabilities to full New Small Wheel area and make it cope with beyond design luminosity rates, up to $15 \mathrm{kHz} / \mathrm{cm}^{2}$

Will replace the present Small Wheel, not designed to exceed $10^{34} \mathrm{~cm}^{-2} \mathrm{~s}^{-1}$

Will operate up to HL-LHC luminosity (Phase-2)

Need fast, precise, and high radiation resistant detectors

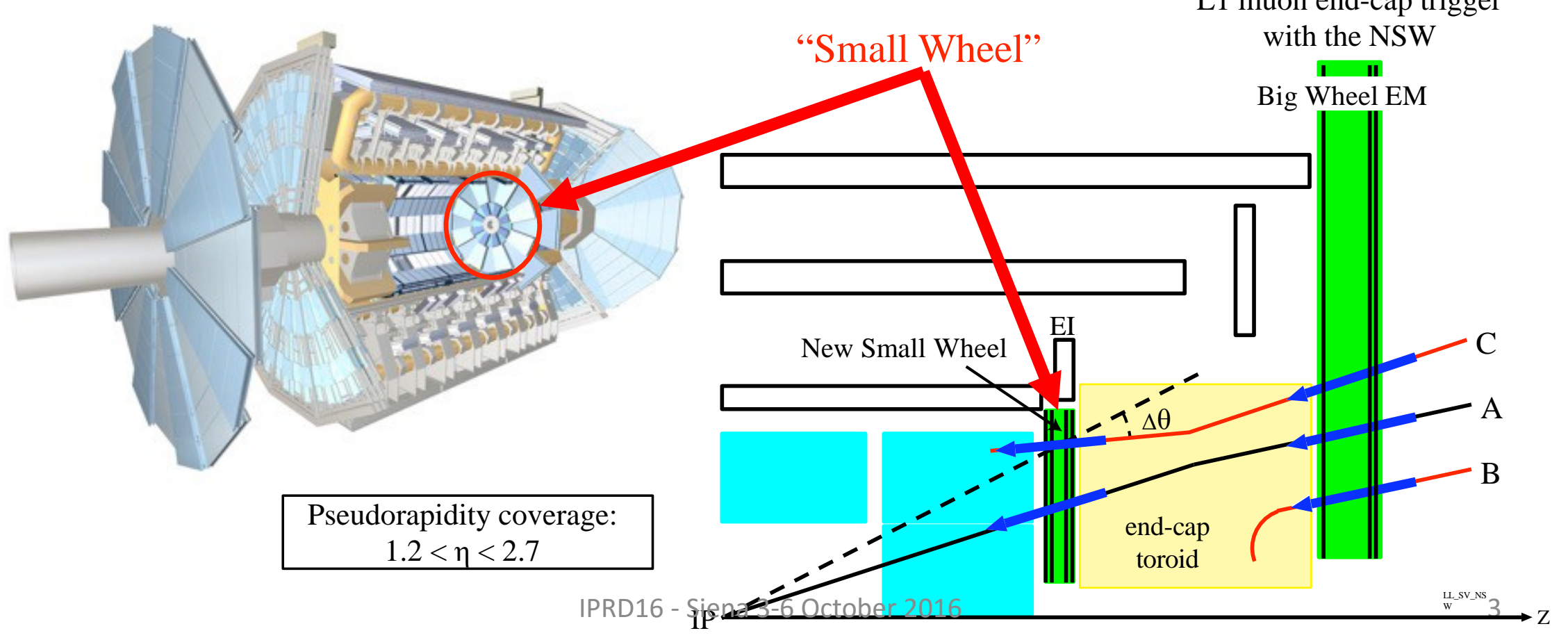
with the NSW

Big Wheel EM

Pseudorapidity coverage: 


\section{New Small Wheel detector technologies}

\section{Combination of sTGC and MicroMegas detector planes}

\section{Small Strips TGC (sTGC)}

primary trigger detector

- Bunch ID with good timing resolution

- Online track vector

with $<1$ mrad angle resolution

- pads: region of interest

- $\quad$ strips: track info (strip pitch $3.2 \mathrm{~mm}$ )

- wire groups: coarse azimuthal coordinate

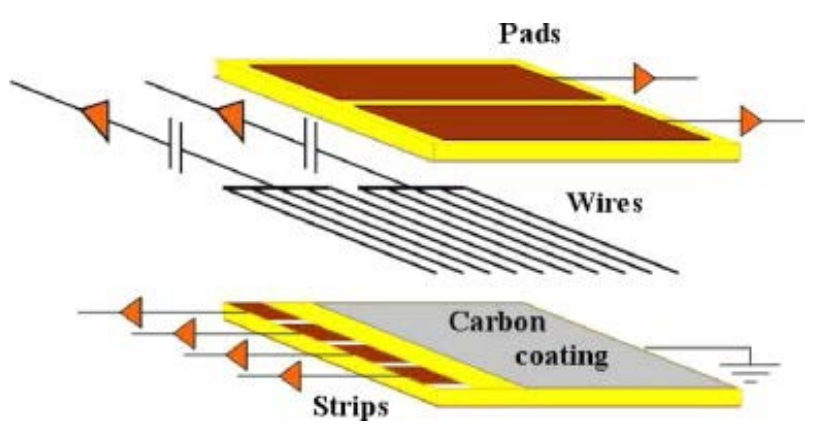

\section{MicroMegas (MM)}

primary precision tracker

- Good Spatial resolution $<100 \mu \mathrm{m}$

- Good track separation (0.4 mm readout granularity)

- Resistive anode strips $\rightarrow$ suppress discharge influence on efficiency

- Provide also online segments for trigger

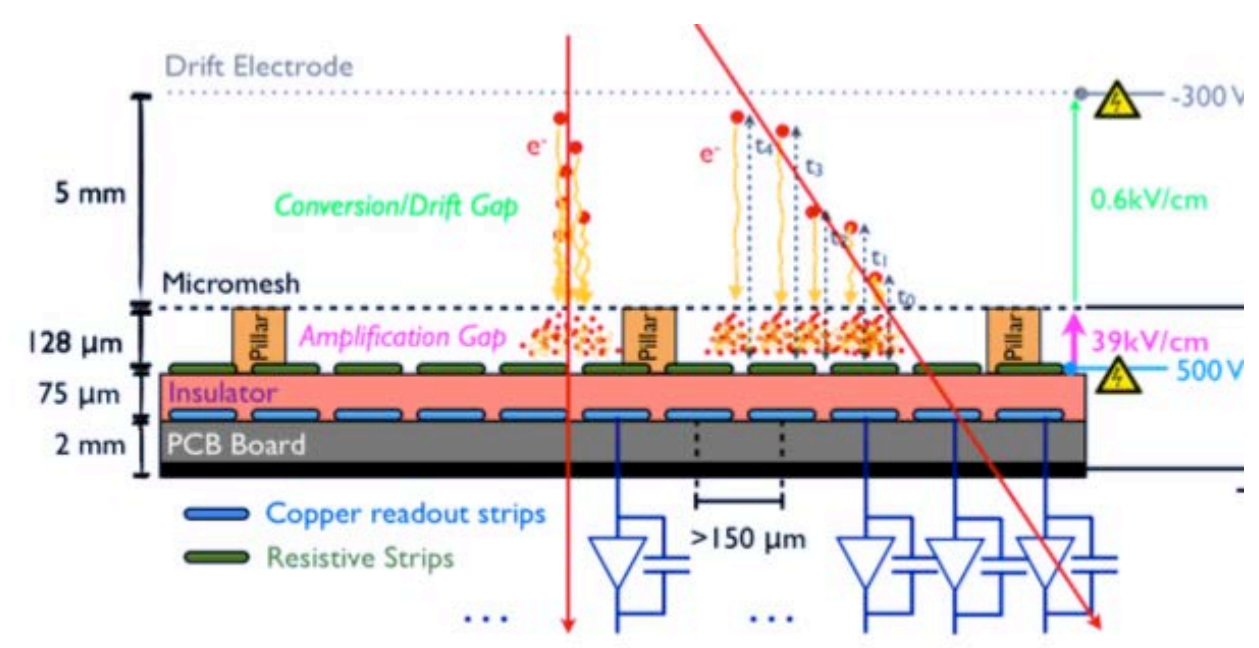

- Common front-end ASIC: VMM second prototype under tests 


\section{New Small Wheel layout}

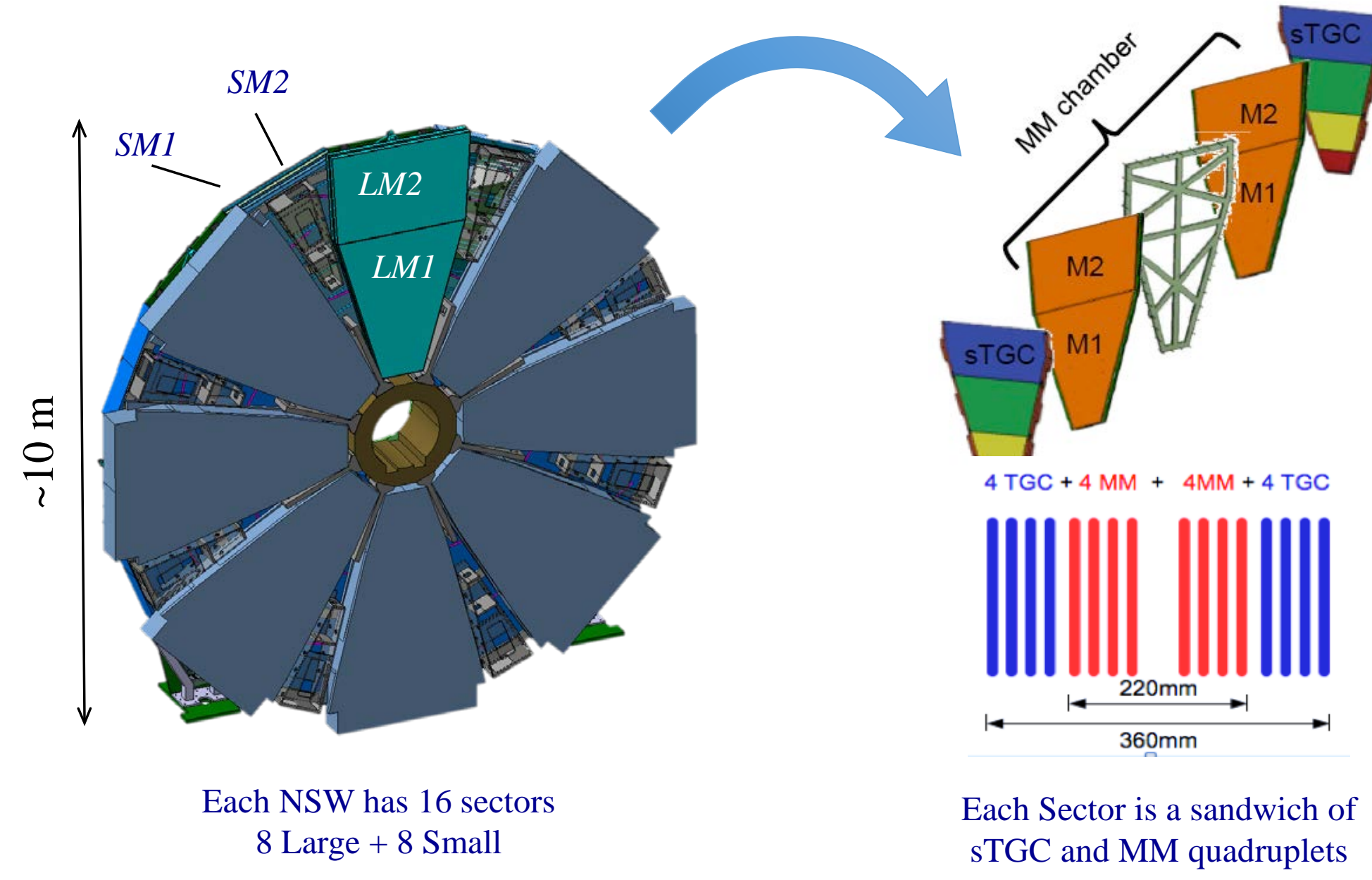




\section{sTGC construction sites}

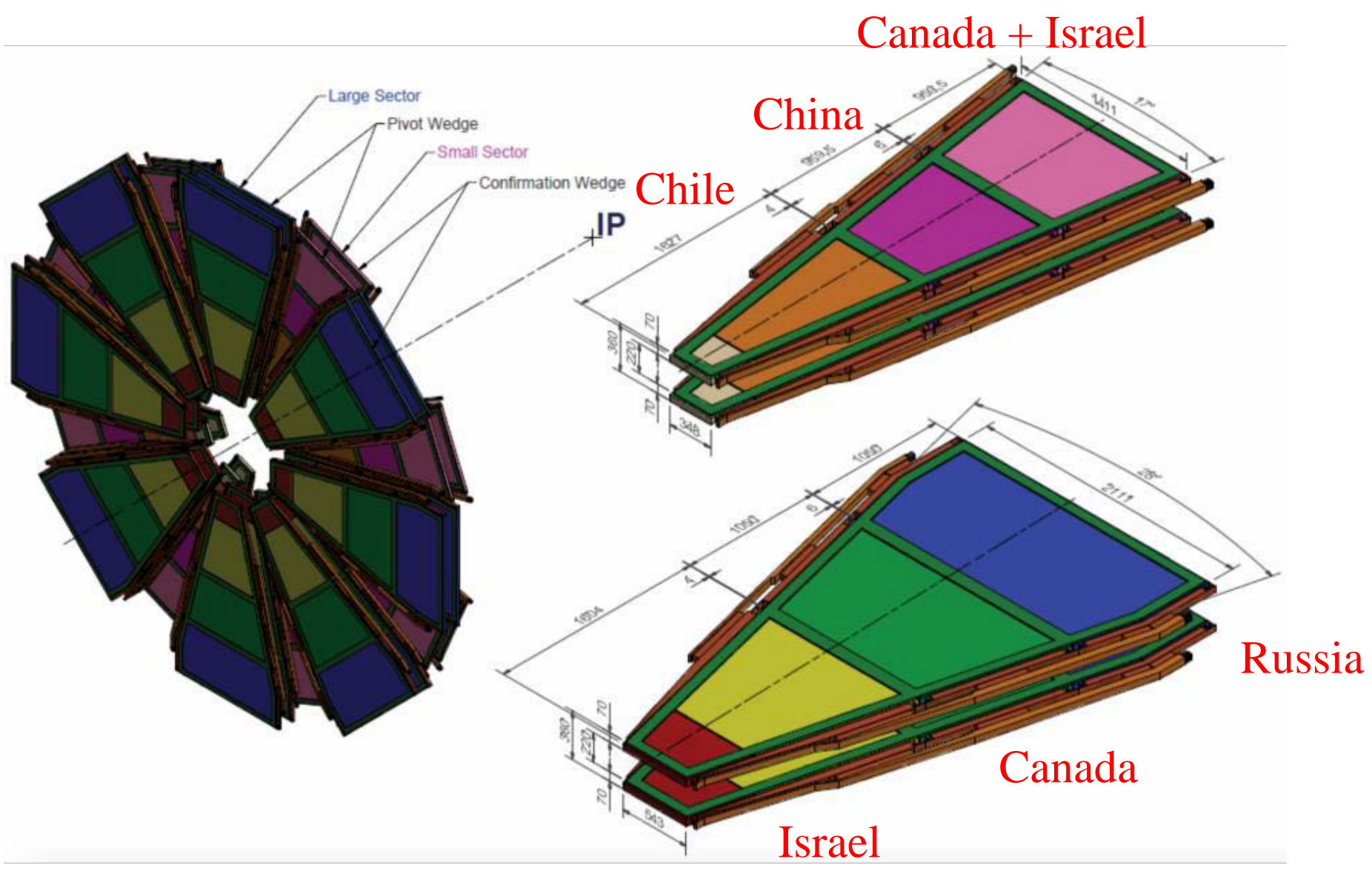




\section{sTGC structure}

- The basic sTGC structure consists of a grid of gold-plated tungsten wires sandwiched between two resistive cathode planes at a distance of $1.4 \mathrm{~mm}$ from the wire plane

- The precision cathode plane has strips with a $3.2 \mathrm{~mm}$ pitch for precision readout relative to a precision brass insert outside the chamber, and the cathode plane on the other side has the pads for triggering

- The gap is provided using $1.4 \mathrm{~mm} \pm 20 \mu \mathrm{m}$ precision frames glued to the cathode boards

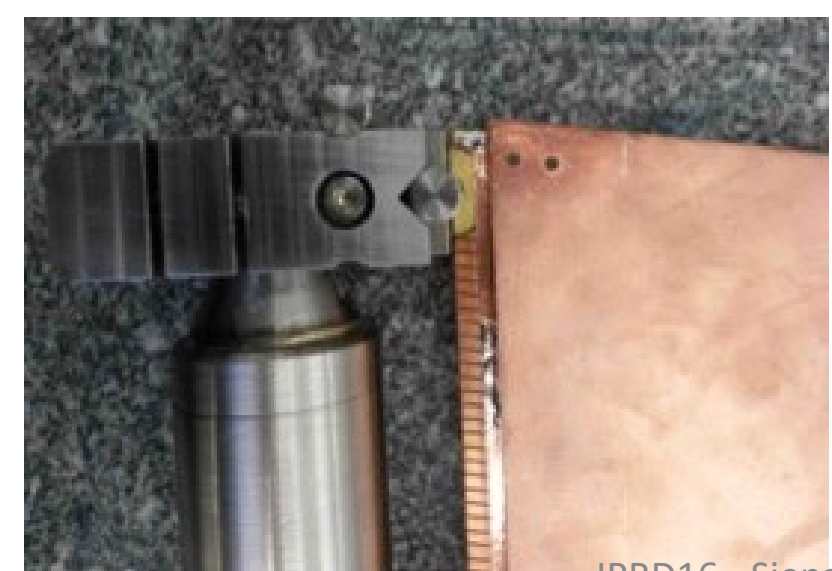

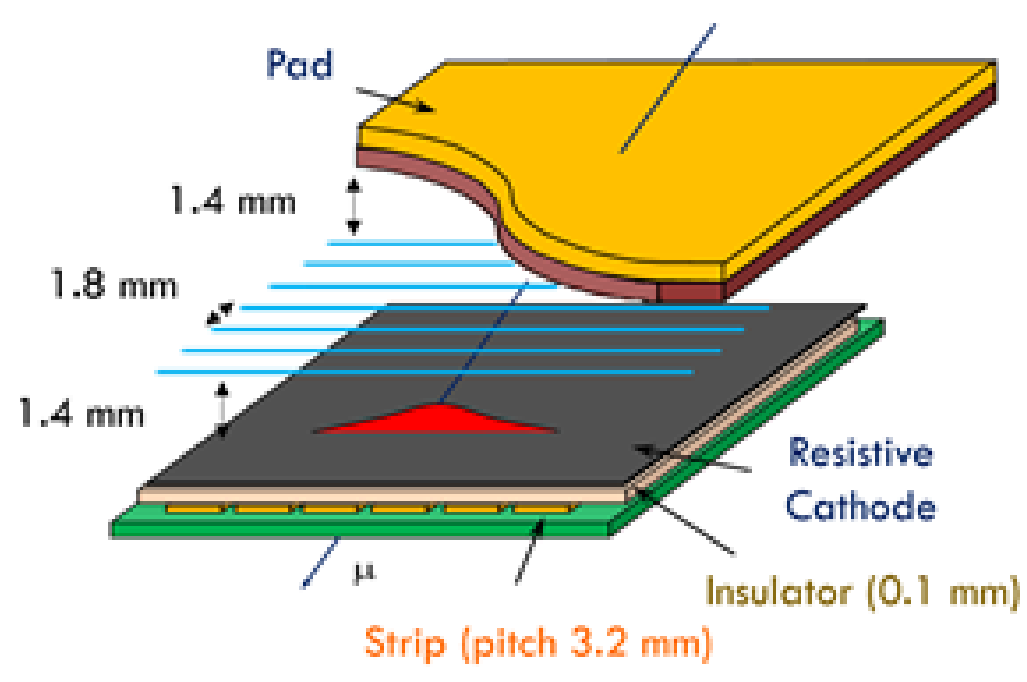

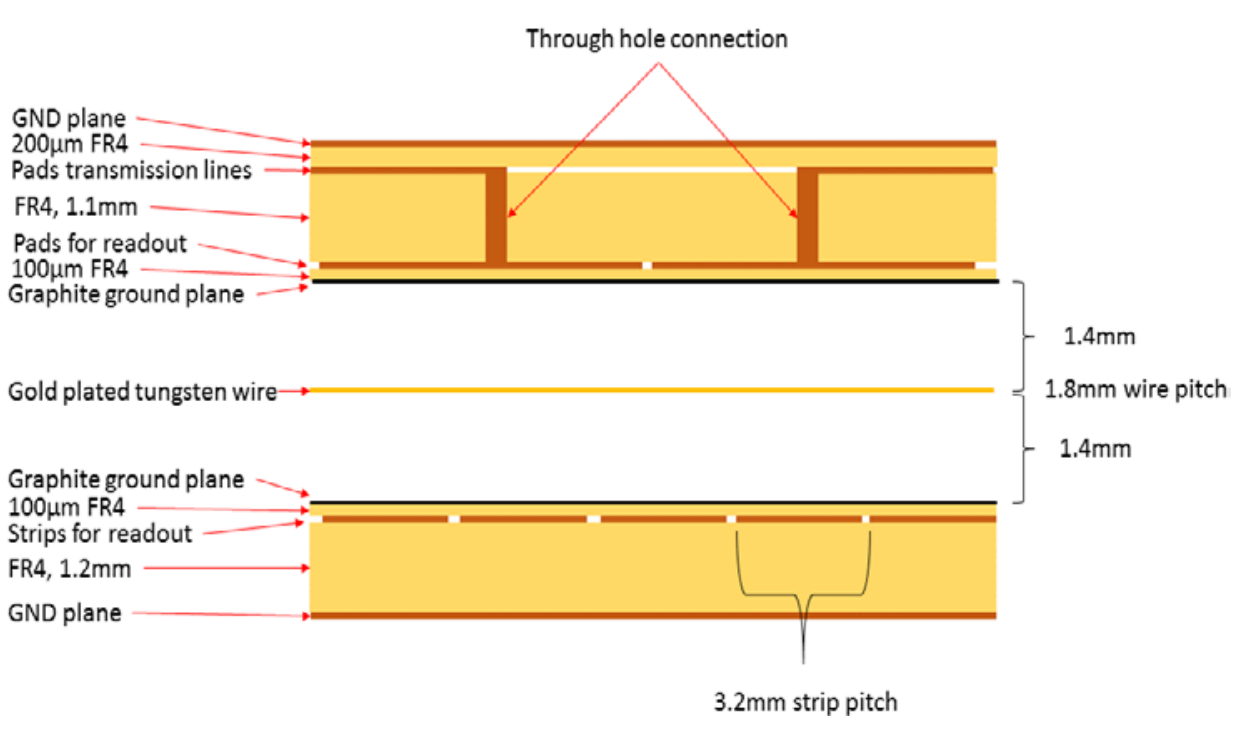




\section{sTGC structure}

- Pad readout provide fast pre-trigger to determine the strip to be read

- Precision strips for precision muon tracking reconstruction at level of $100 \mu \mathrm{m}$

- High efficiency at high background rate

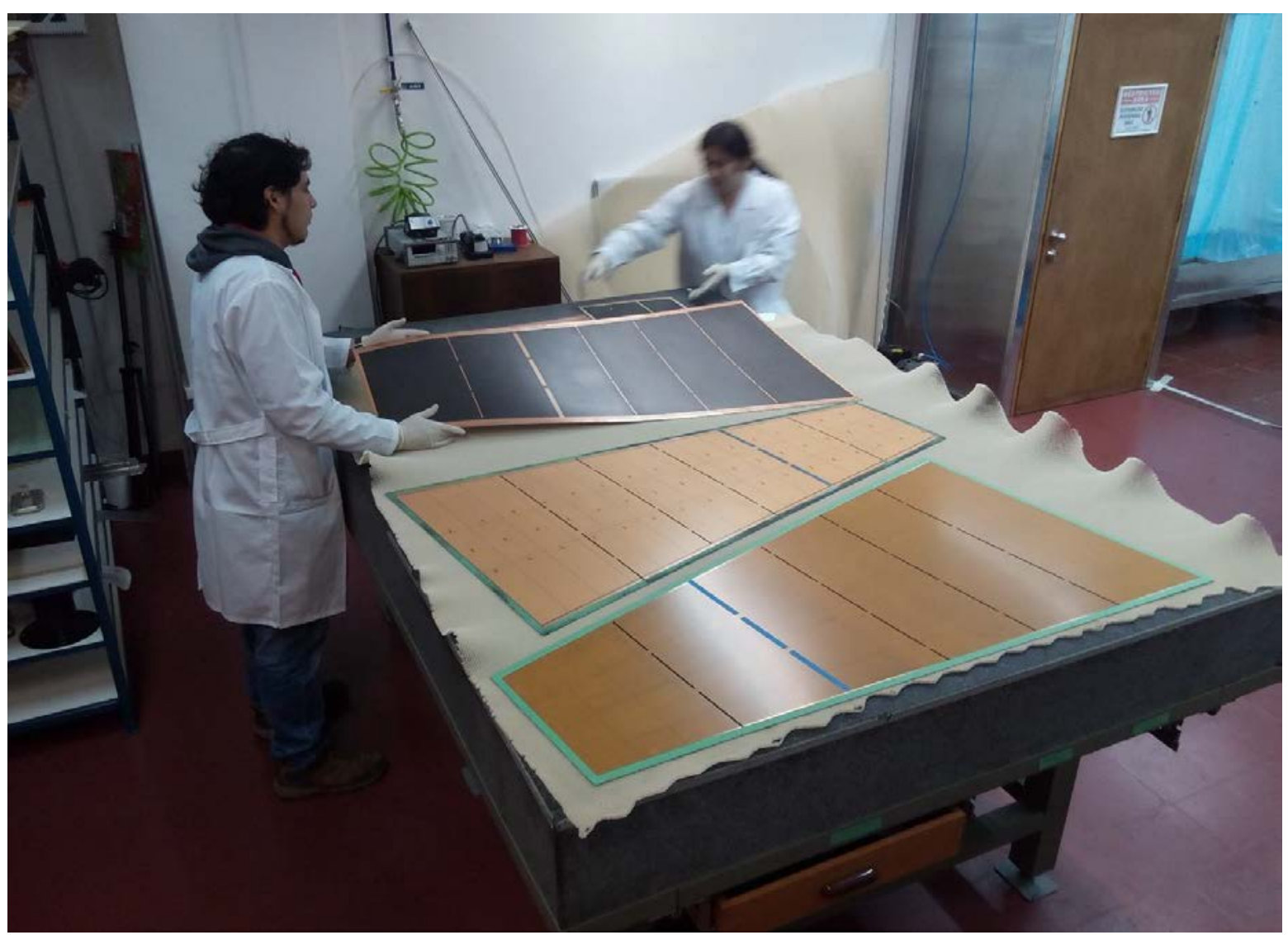




\section{sTGC working conditions}

- sTGC chambers are working on n-Pentane/CO2 (45\% / 55\%) gas mixture

- This mixture has three main properties:

- High gain

- Quenching of photons

- Clean the chamber

- Nominal operational voltage $-2800 \mathrm{~V}$

- The cathode plane is made by the resistive layer of graphite with a surface resistivity of $\sim 100 \mathrm{k} \Omega / \square(200 \mathrm{k} \Omega / \square$ for outer chambers $)$

- All quadruplets have trapezoidal shapes with surface area up to $2 \mathrm{~m}^{2}$ 


\section{Module-0s}
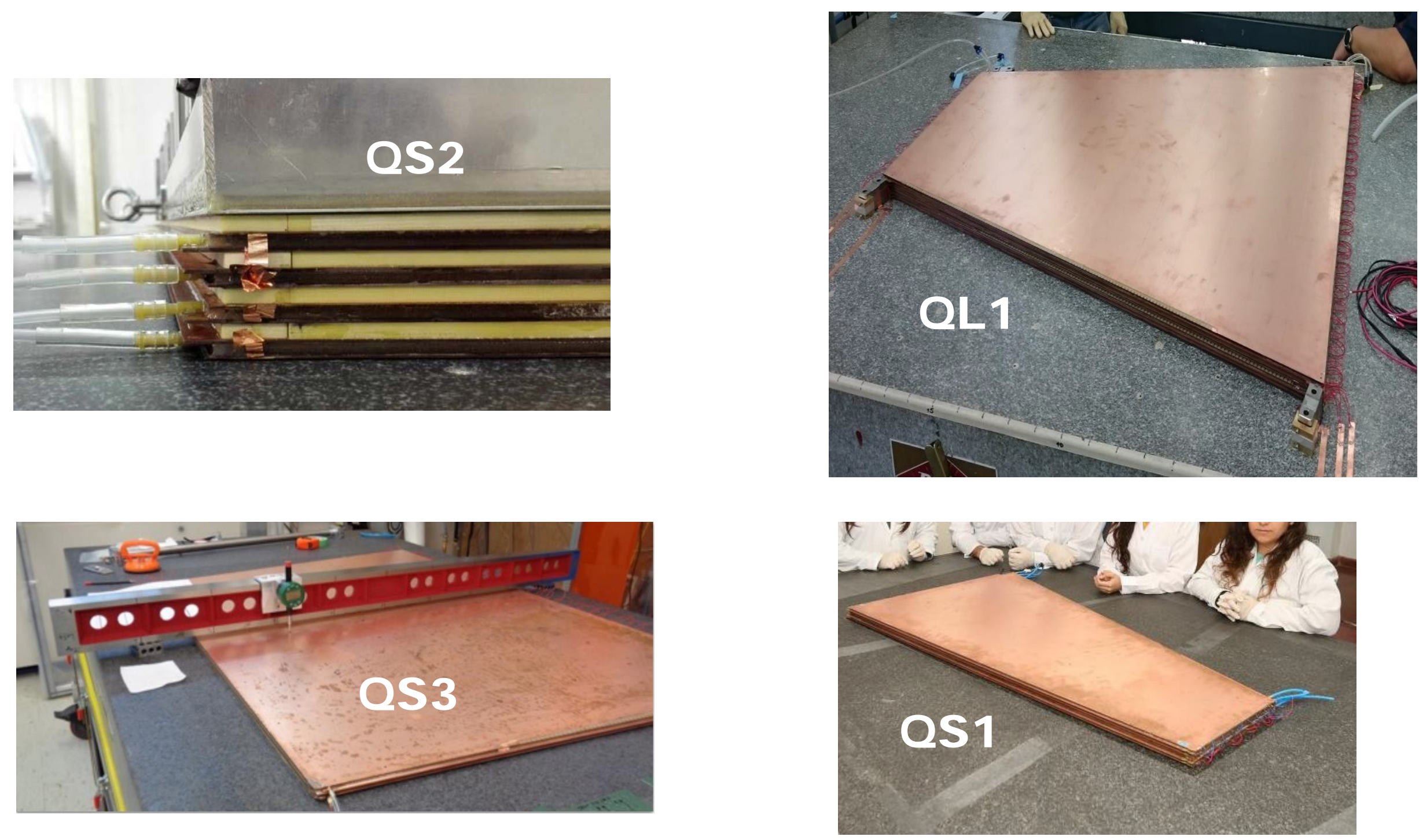


\section{Gain uniformity test}

Gain uniformity test has been performed using Mini-Xray gun by the scanning of whole surface of the chamber

Measurements

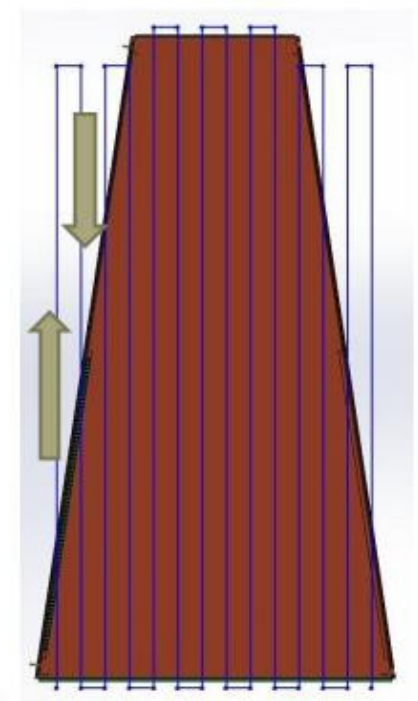

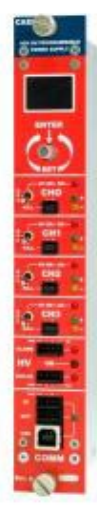

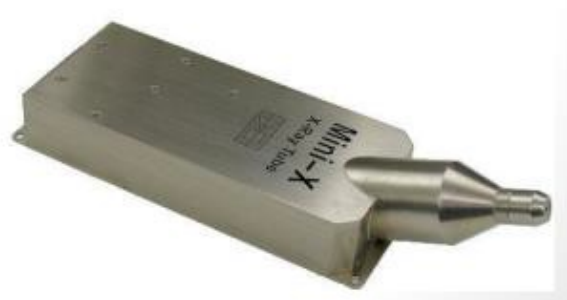

Hot spot ht

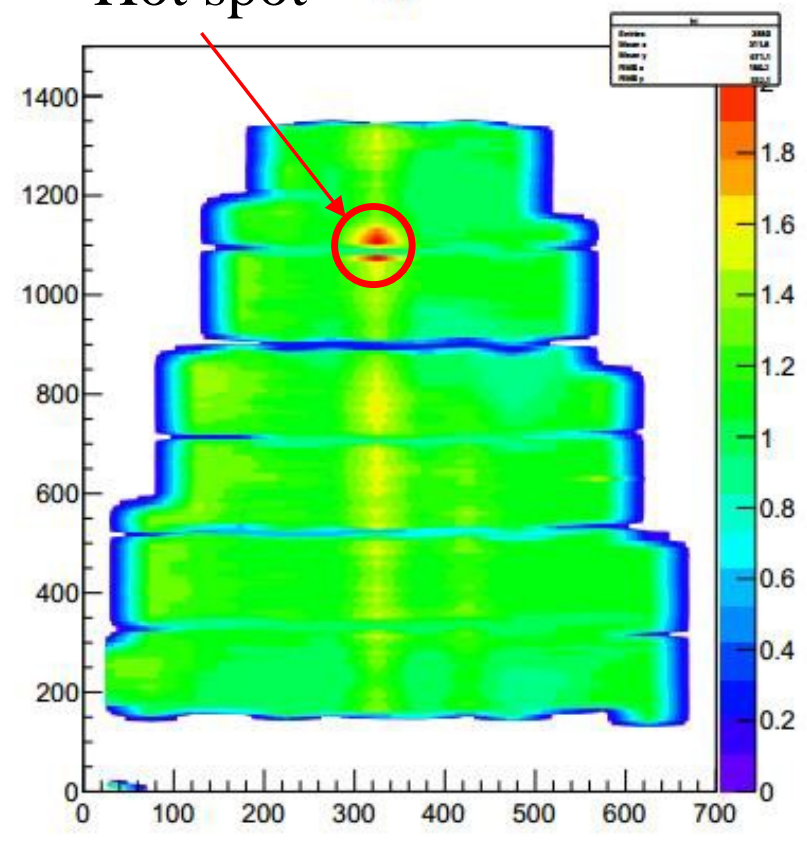




\section{Position resolution measurement at Fermilab}

- Position resolution was measured using $32 \mathrm{GeV}$ pion beam at Femilab

- The results shown a position resolution to be better than $\pm 50 \mu \mathrm{m}$ comparing to an external pixel telescope for different position scan in $\mathrm{X}-\mathrm{Y}$ $(\mathrm{A}, \mathrm{B}, \mathrm{C}, \mathrm{D}, \mathrm{E}, \mathrm{F})$ in all four layers
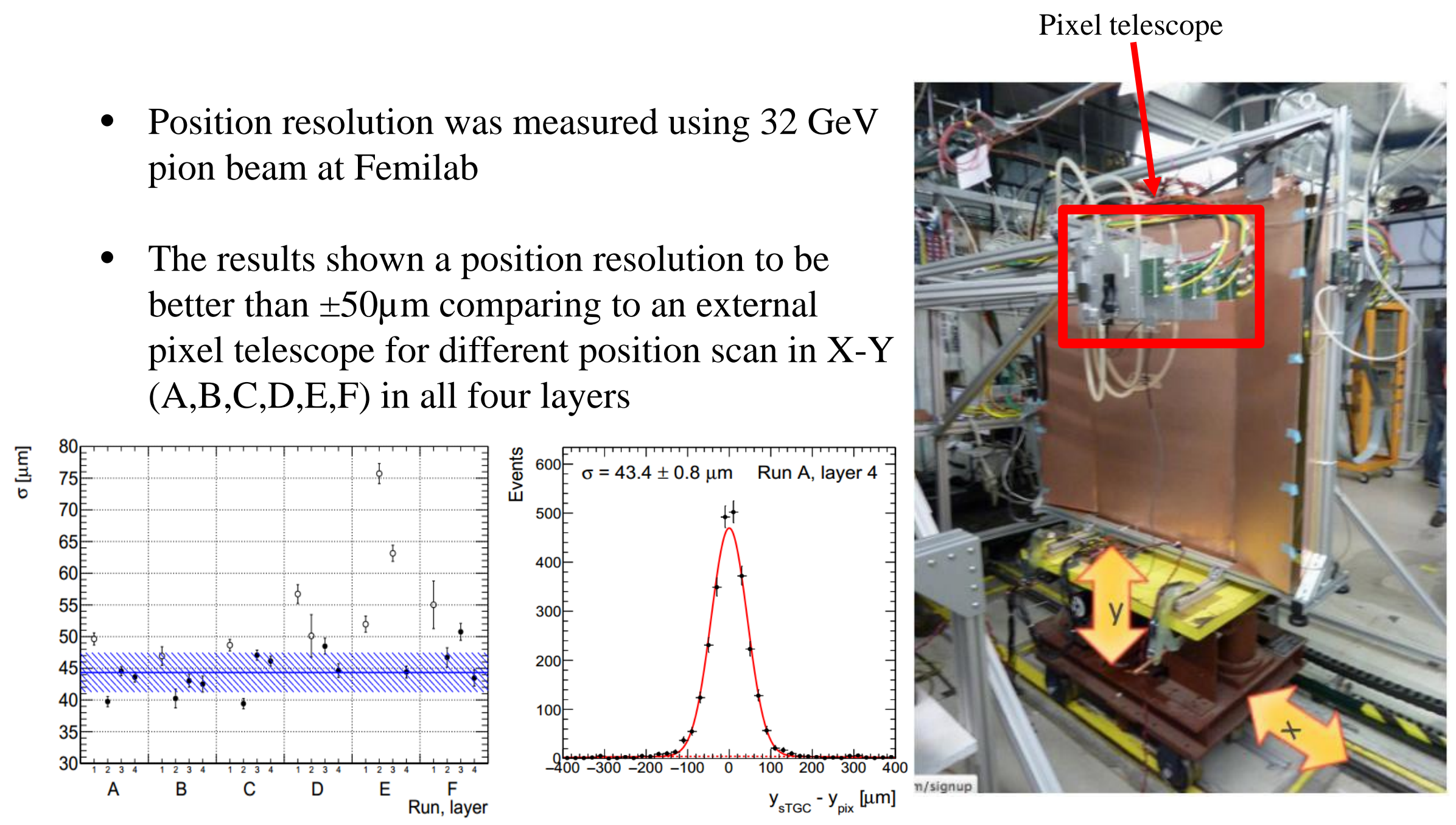


\section{MicroMegas construction sites}
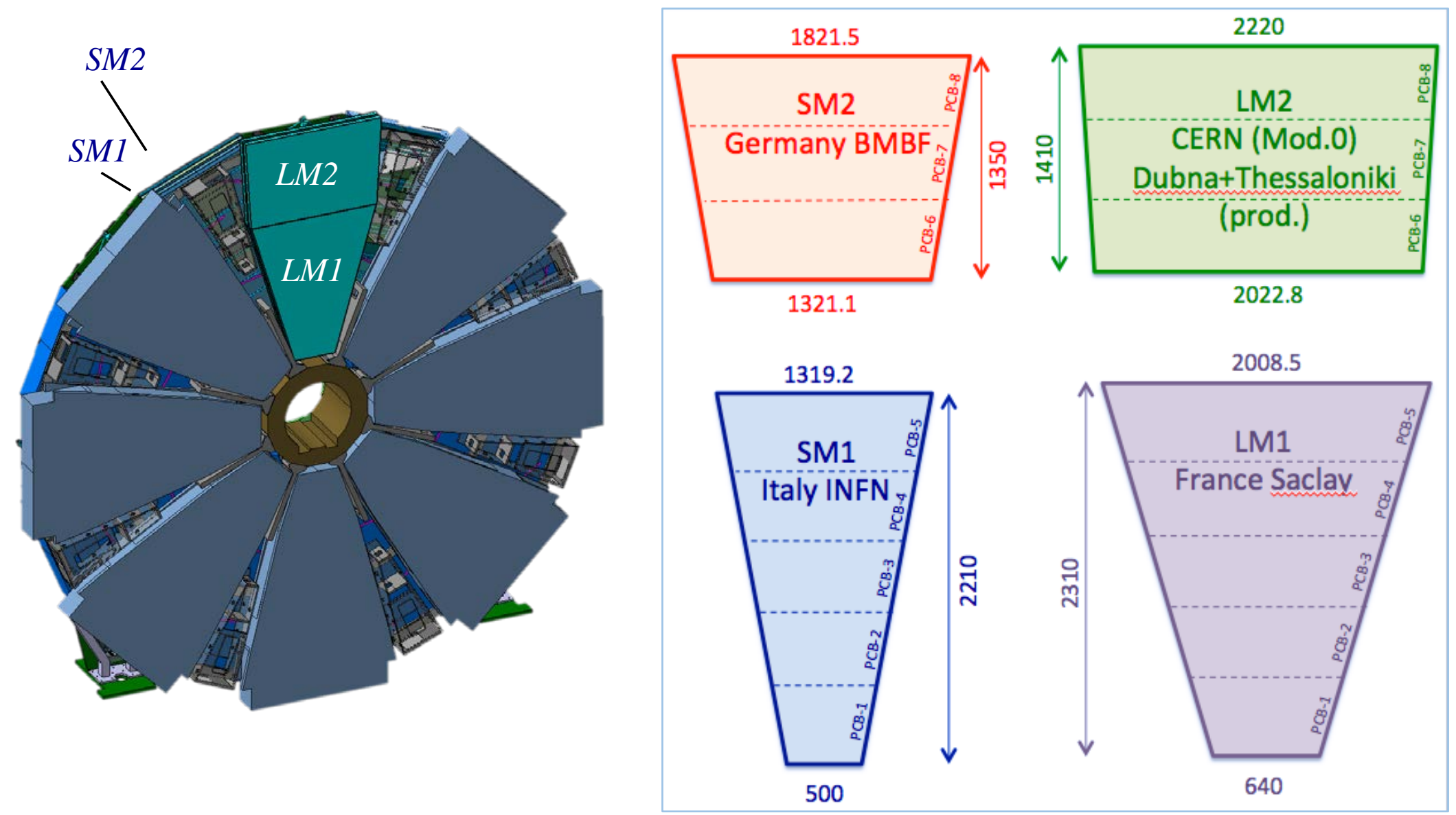


\section{MicroMegas construction: Read-out boards and resistive strips}
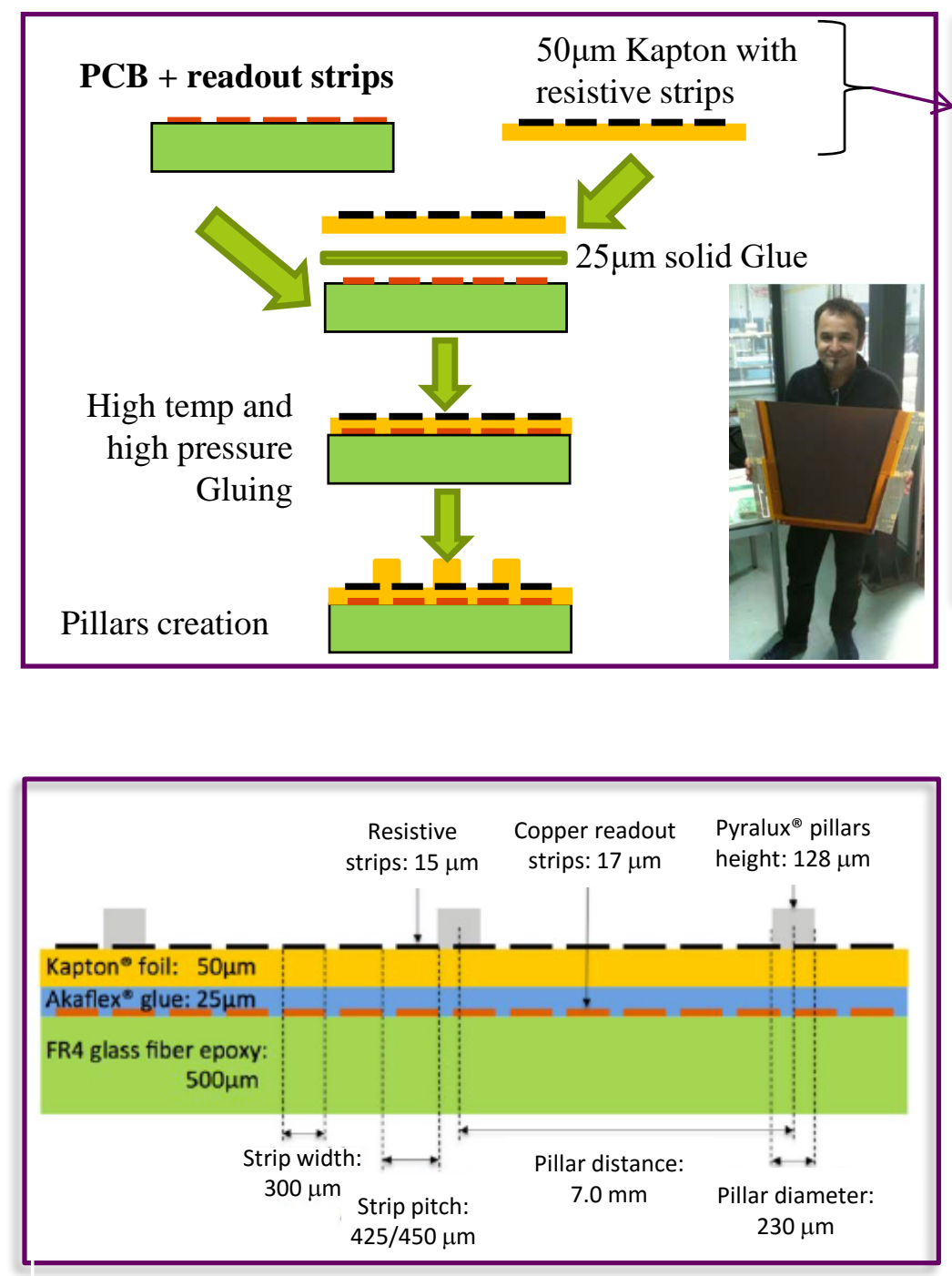

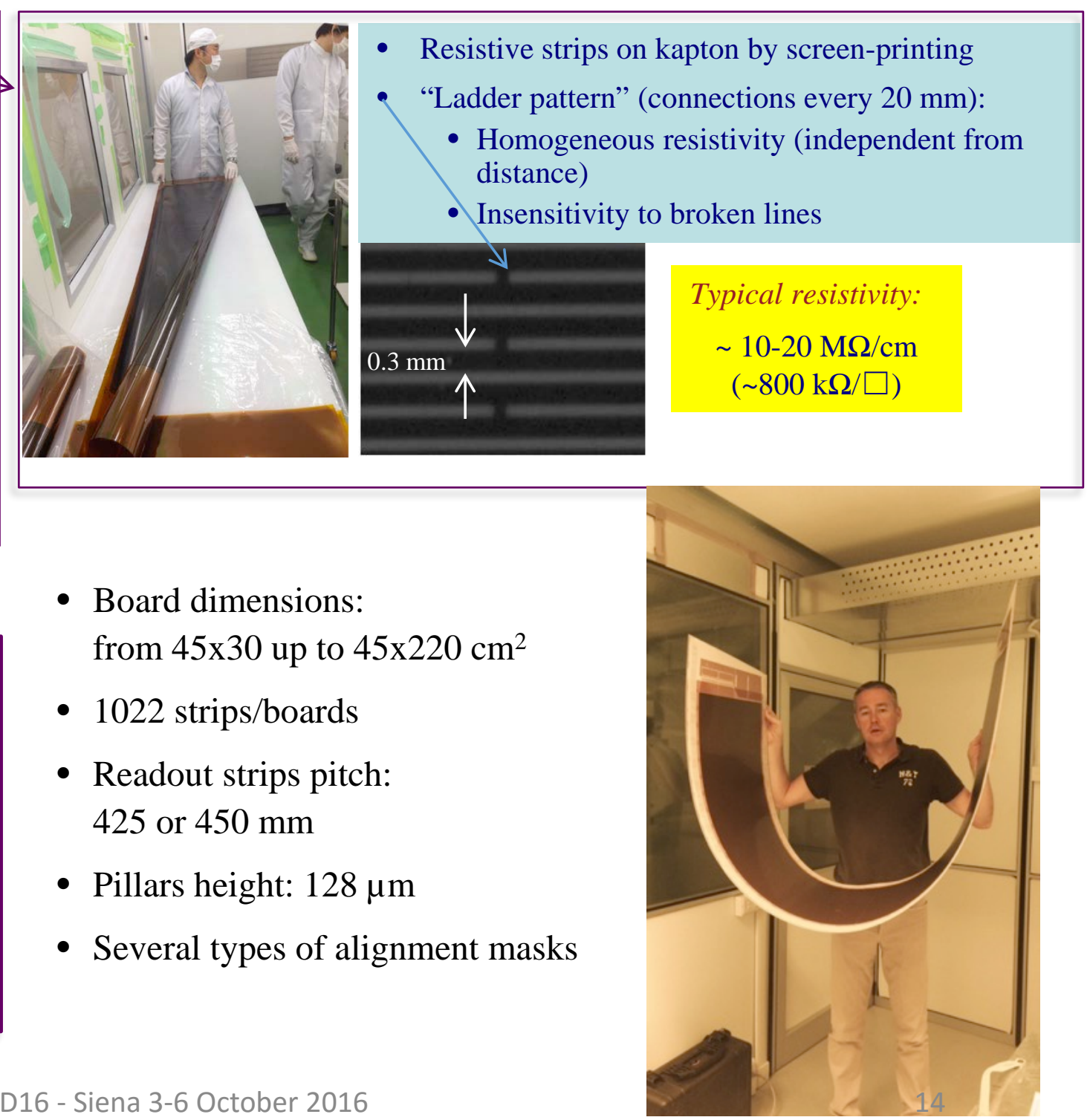




\section{MicroMegas construction: drift panel}

Similar construction Concept as for the readout panels

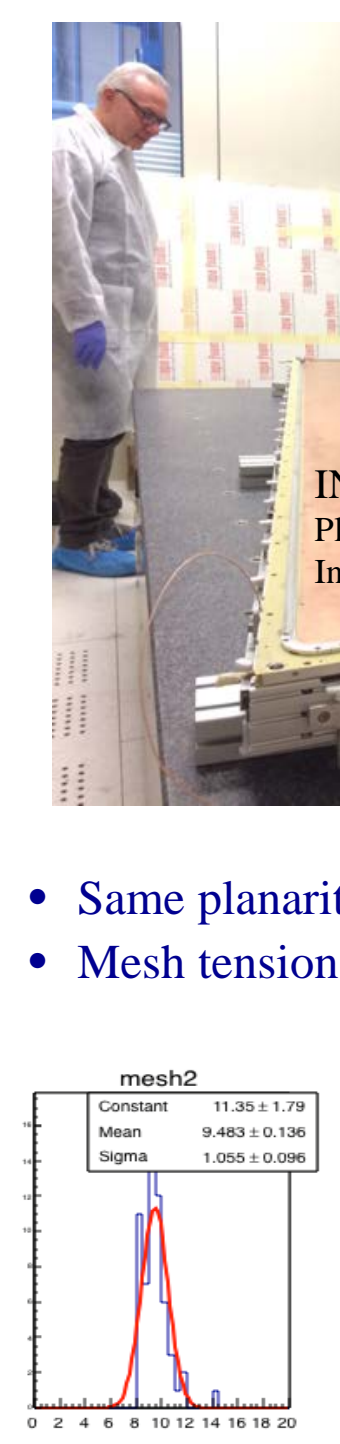

INFN Roma1

Planarity on several panels:

In the range 28 - $39 \mu \mathrm{m}$ RMS

- Same planarity requirements as RO panels

- Mesh tension 10 N/cm - uniformity 10\%
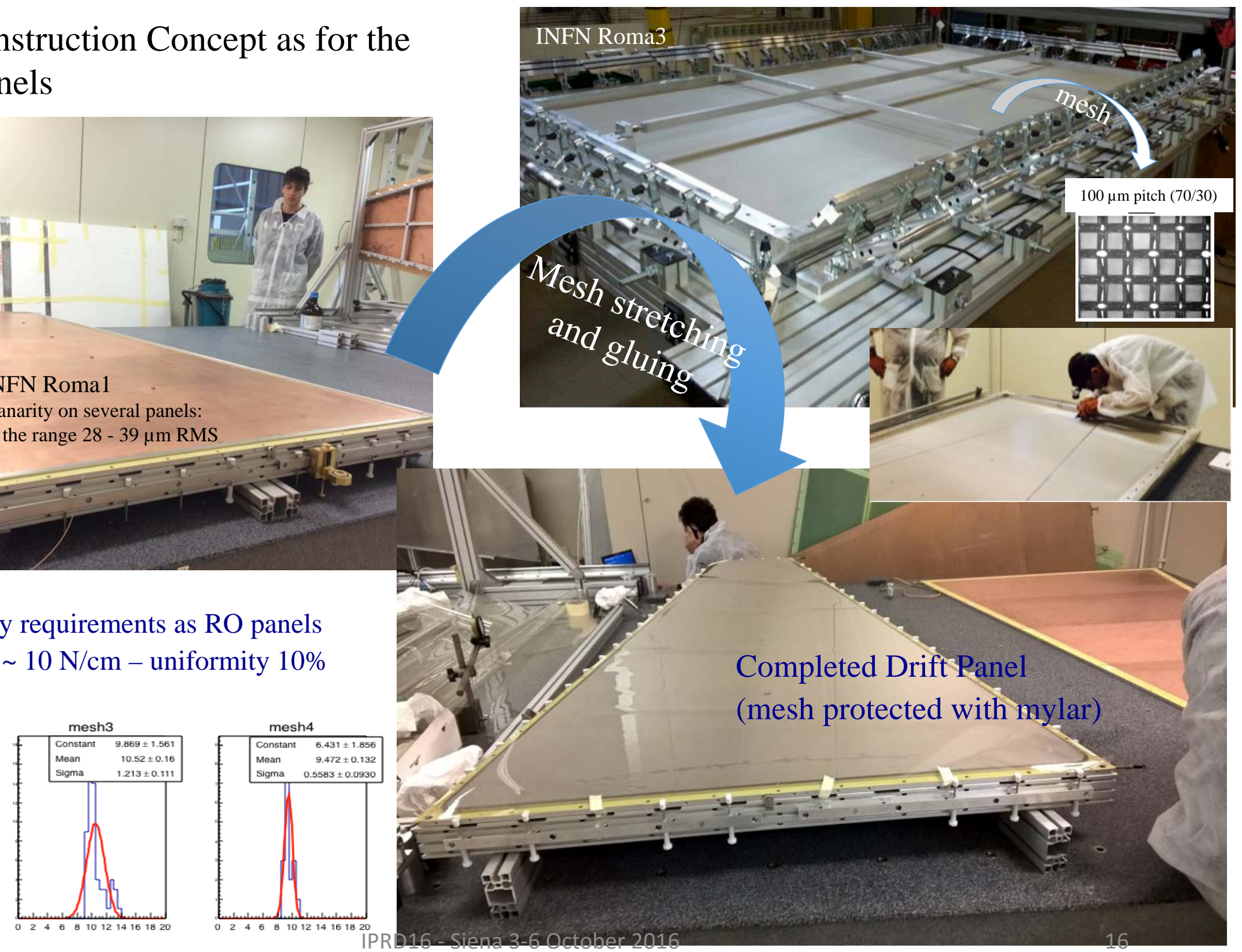


\section{SM1 Module-0 quadruplet assembly}

Crucial: Alignment of the two readout panels at < $60 \mathrm{~mm}$ precision

Vertical Assembly

Alignment of eta Vs Stereo

Readout Panel ensured by

alignment pins

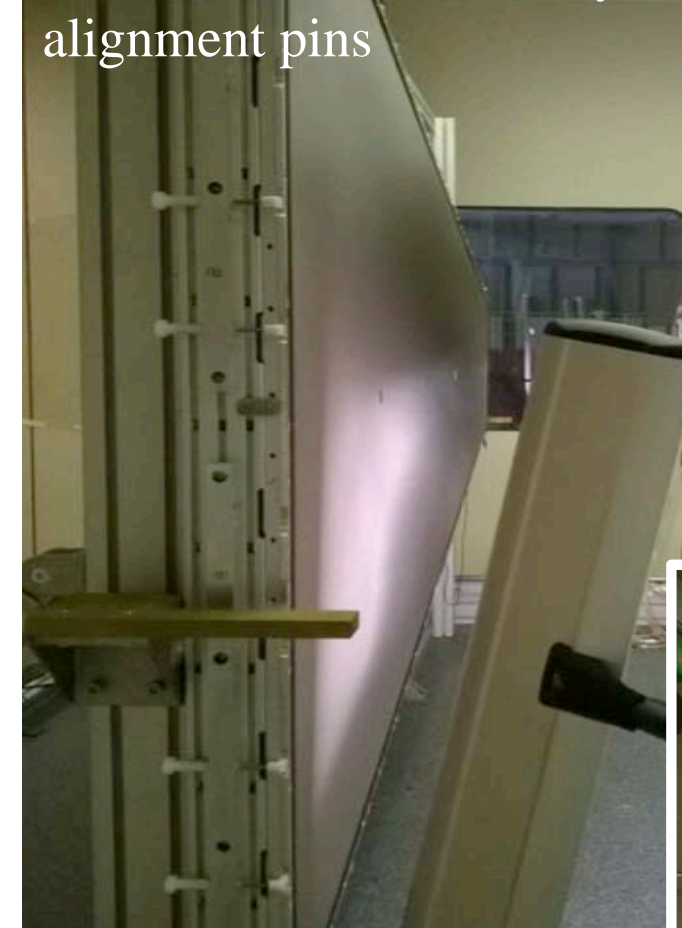

slot alignment bushing

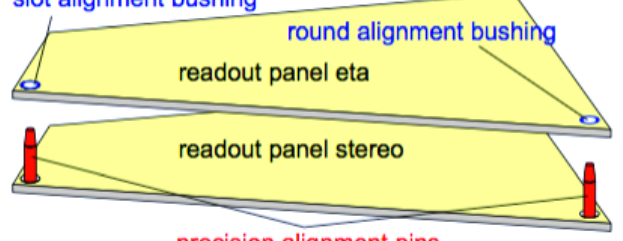

precision alignment pins

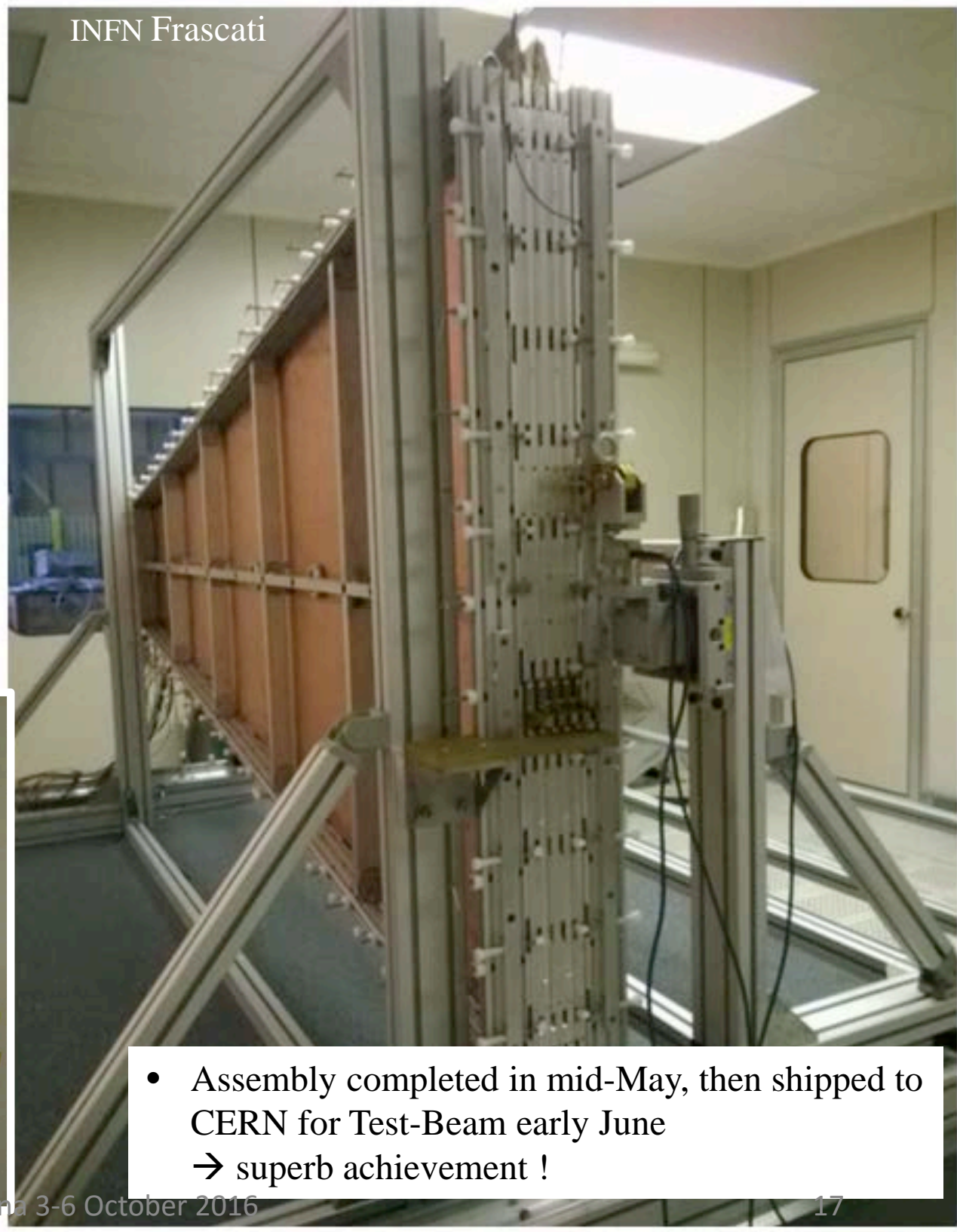




\section{Test beam results at CERN}

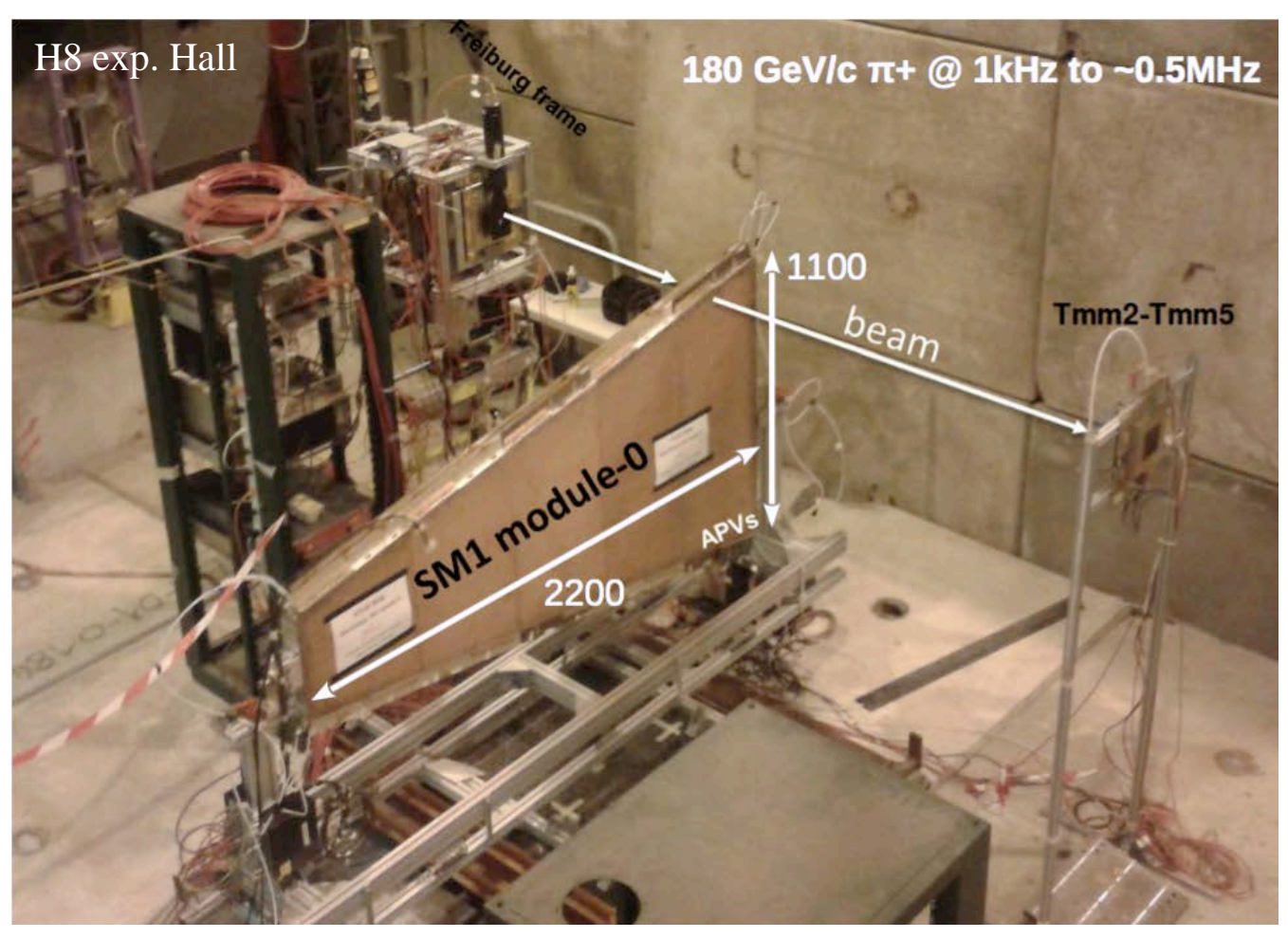

- $180 \mathrm{GeV} / \mathrm{c}$ pion beam

- Scintillators trigger

- Beam spot+trigger $\sim 1 \mathrm{x} 1 \mathrm{~cm}^{2}$

- 5 small MicroMegas chambers with x-y readout (Tmm) used as reference

- SM1 Module-0 on a x-y scanning table

- Ar: $\mathrm{CO}_{2}$ gas mixture (93:7)

- APV25+SRS readout (from RD51) [NOT FINAL NSW electronics]

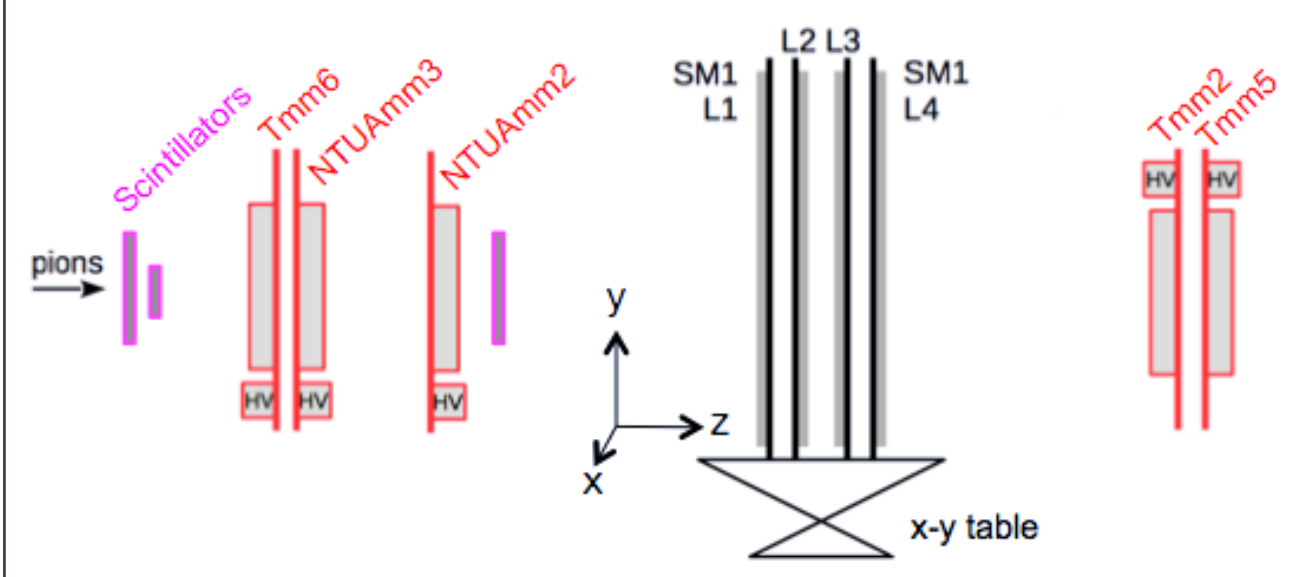

SM1 quadruplet:

- $425 \mu \mathrm{m}$ strip pitch

- L1 \& L2 vertical strips (eta),

- L3 \& L4 $\pm 1.5^{\circ}$ w.r.t. vertical axis (stereo)

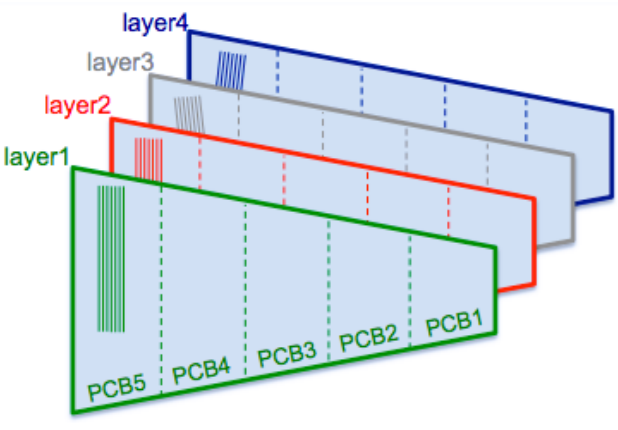




\section{Test beam results at CERN}

- Perpendicular incident beam on PCB5 = longest strips

- Nominal High voltage settings:

$$
\begin{aligned}
& \mathrm{HV} \_ \text {ampl }=570 \mathrm{~V}(\mathrm{E}=44 \mathrm{kV} / \mathrm{cm}) \\
& \mathrm{HV} \_ \text {drift }=300 \mathrm{~V}(\mathrm{E}=600 \mathrm{~V} / \mathrm{cm})
\end{aligned}
$$

- Ar/CO 2 93/7 @ 20 l/hour
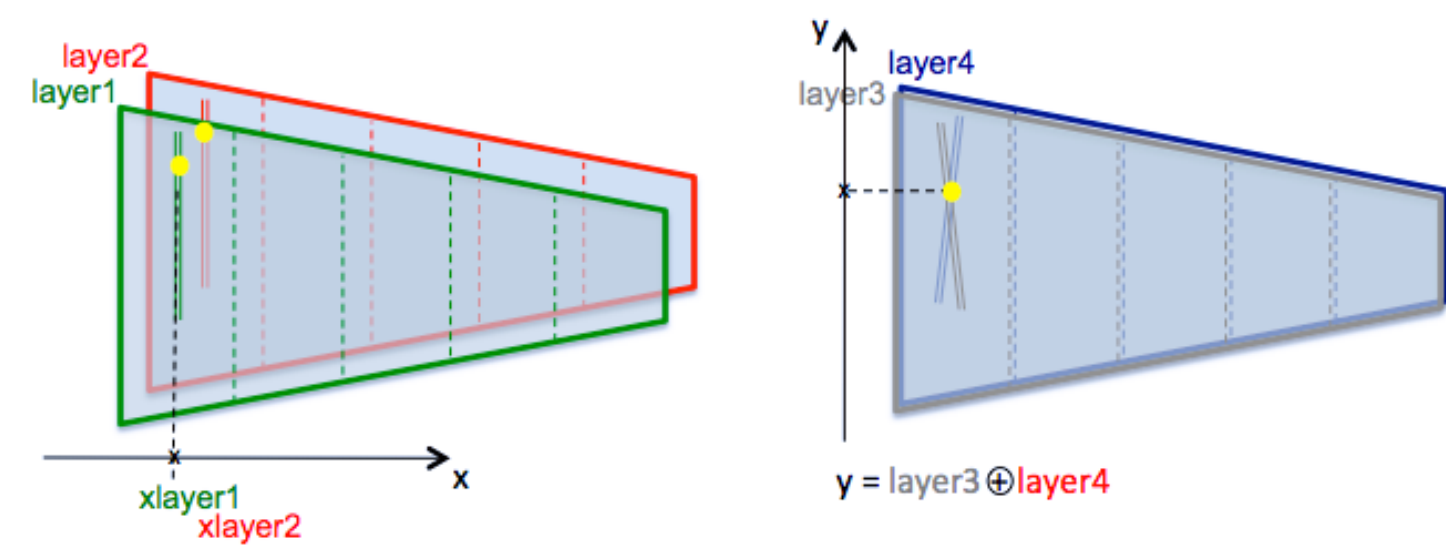

y = layer $3 \oplus$ layer4

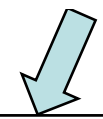

Preliminary result: Spatial Resolution of the precision coordinate ATLAS NSW Preliminary

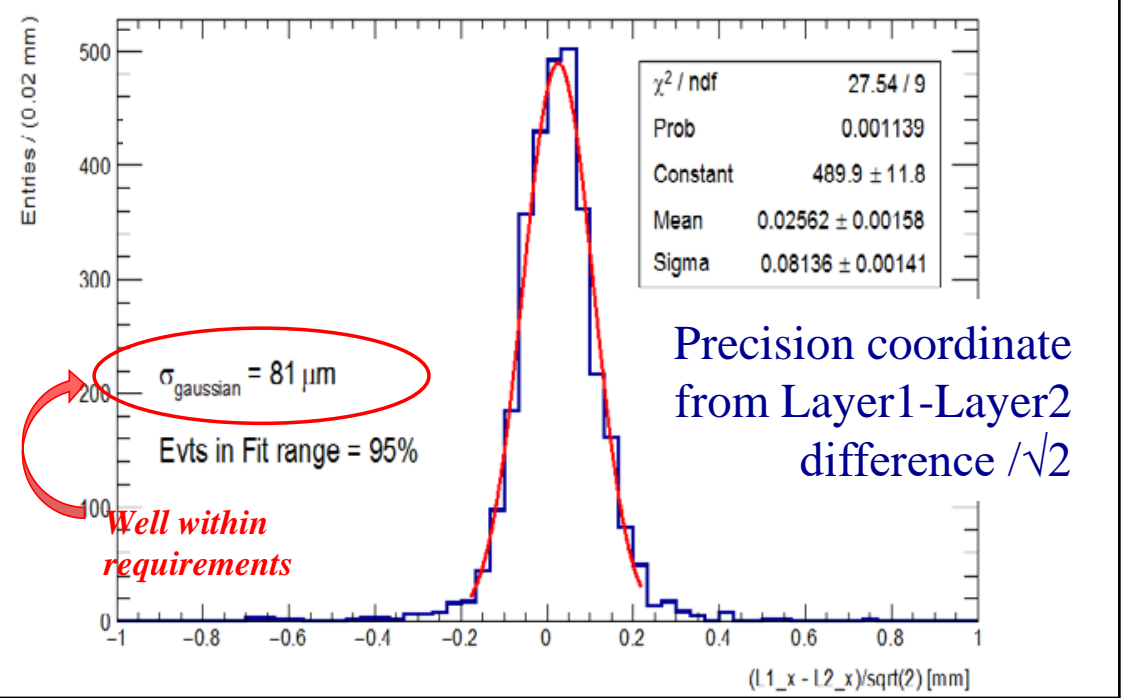

Preliminary result: Spatial Resolution of the second coordinate.

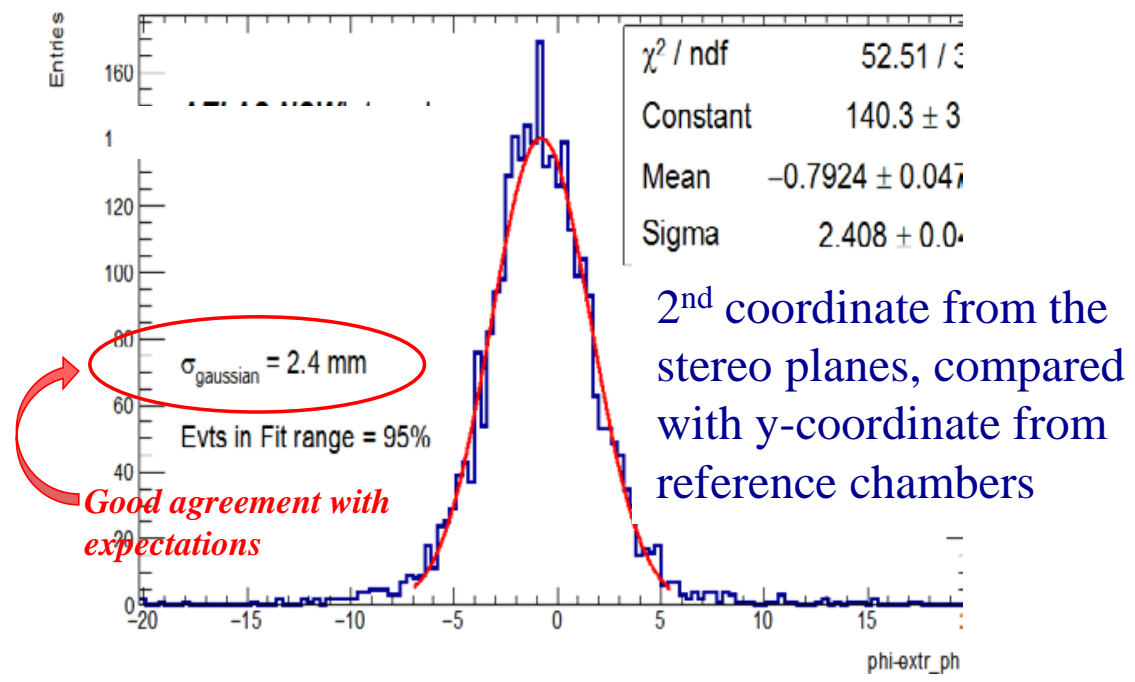




\section{Test beam results at CERN}

Cluster efficiency Vs Amplification HV for Layer1

- Cluster efficiency: presence of a cluster for any reference track

- Track-based efficiency: one cluster within given distance from the reference track impact on SM1

- Turn-on curve saturate at a cluster efficiency very close to $100 \%$

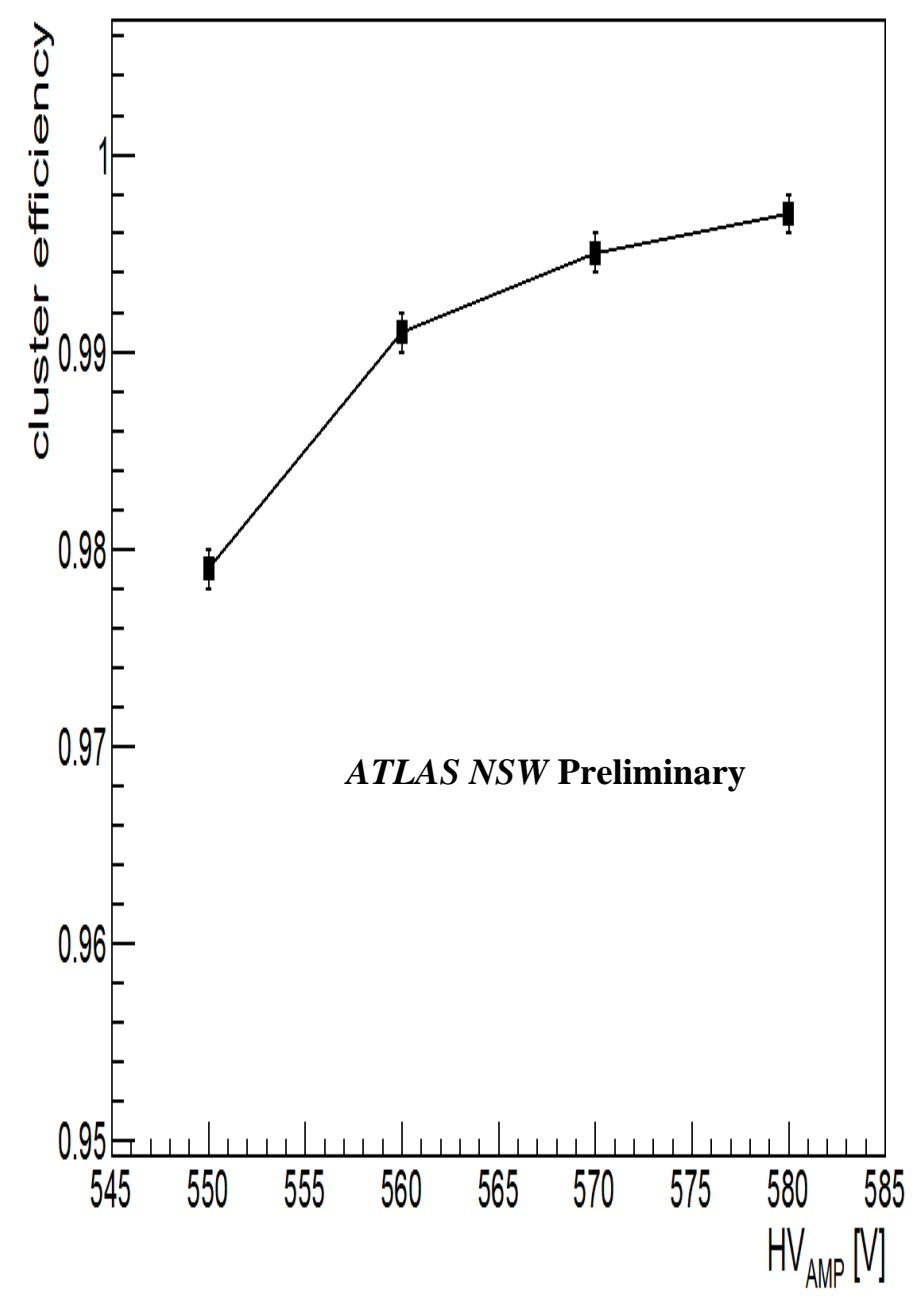




\section{Conclusions}

- The ATLAS NSW Upgrade will enable the Muon Spectrometer to retain its excellent performance also beyond design luminosity and for the HL-LHC phase

- Large size resistive MicroMegas will be employed for the first time in HEP experiments

Thanks to great effort from all collaboration groups from different universities and institutes from different countries

- sTGC:

Canada, Chile, China, Israel, Russia

- MicroMegas:

France, Germany, Greece, Italy, Russia

the project is well advanced and will be ready for LS-2 


\section{Back-up slides}




\section{ATLAS New Small Wheel}

- Help rejecting background in the forward region, allowing to keep low $\mathrm{p}_{\text {т }}$ thresholds in the trigger at high luminosities, to study interesting physics processes with low рт muons (for instance $125 \mathrm{GeV}$ Higgs)

- $\quad$ The current SW can't cope with rates up to $15 \mathrm{kHz} / \mathrm{cm}^{2}$; degradation in tracking efficiency and resolution.
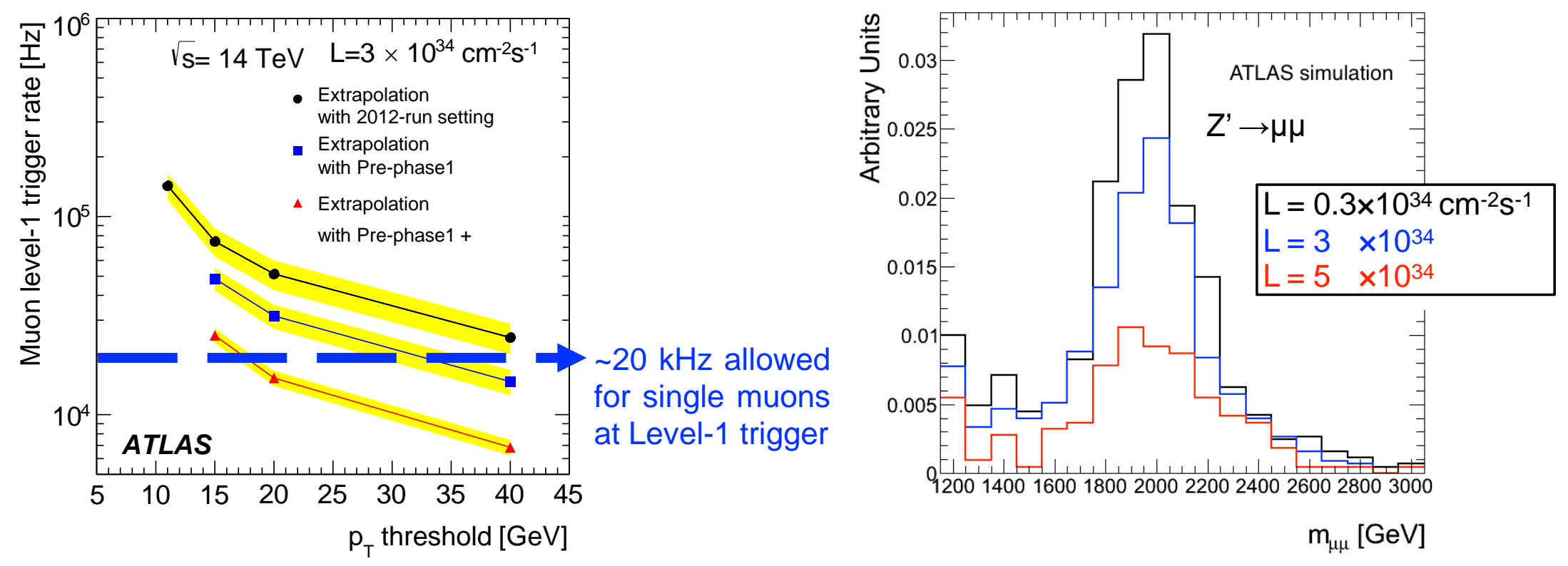


\section{Test beam results at CERN}

- Efficiency was measured at CERN SPS H8 channel using $130 \mathrm{GeV}$ muon beam about $8 \mathrm{~cm}$ diameter

- It was determined that the detector efficiency is $\sim 100 \%$

- When particle cross is between two adjacent pads, charge is shared between them

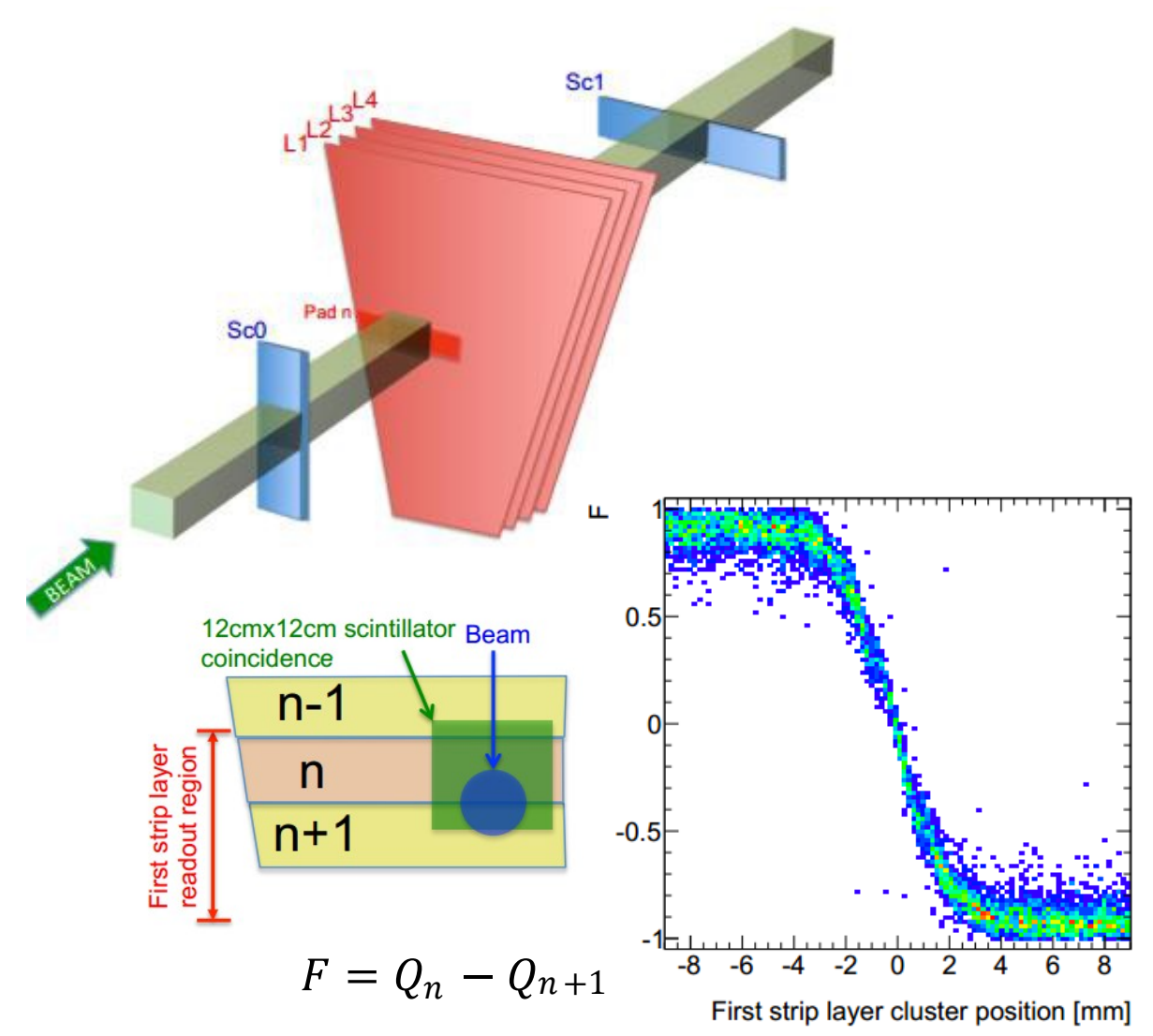

